\title{
The Benefits of Nuts for Cancer Prevention
}

\author{
PAMELA HALLQUIST VIALE, RN, MS, CNS, ANP
}

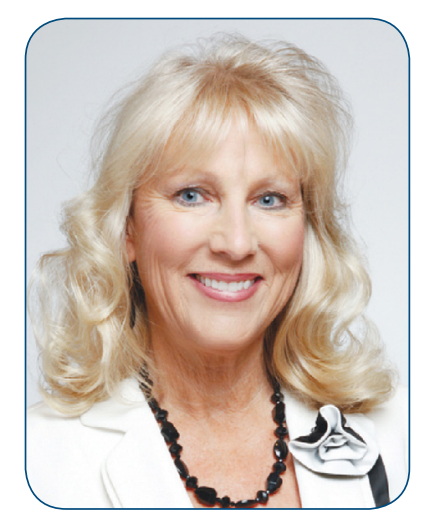

J Adv Pract Oncol 2019;10(2):102-103 https://doi.org/10.6004/jadpro.2019.10.2.1 (c) 2019 Harborside ${ }^{\text {TM }}$

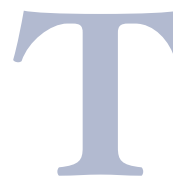

he American Cancer Society estimates 101,420 new cases of colon cancer and 44,180 new cases of rectal cancer in the United States this year (American Cancer Society, 2019). Colorectal cancer remains the third leading cause of death from cancer in both sexes, with approximately 51,020 deaths expected this year (American Cancer Society, 2019). And disturbingly, although the overall death rate has dropped, the death rate for younger people (aged 55 or younger) has increased $1 \%$ per year from 2007 through 2016 (American Cancer Society, 2019).

These statistics are sobering to me, as this is a cancer that is largely preventable through rigorous screening. It is one of the cancers we can successfully do something about. And yet, we still see colorectal cancer. For this reason, I am always interested in data that show variables that may reduce colorectal cancer and death in patients with this disease.

\section{REDUCING INCIDENCE AND DEATH IN PATIENTS WITH COLON CANCER}

Factors affecting the risk of colon cancer recurrence and death vary. However, recent prospective data from observational studies have suggested that diet and lifestyle factors may affect the risk of colon cancer recurrence and death. These factors include type 2 diabetes, obesity, a Western diet, high intake of sugared beverages, and increased glycemic load diets (Fadelu et al., 2018). Mortality in colorectal cancer patients may be affected by elevated plasma C-peptide levels or low insulin-like growth factor-binding protein 1 levels, suggesting an association between energy excess and disease recurrence (Fadelu et al., 2018). The authors of a published study noted that in prospective cohort studies, increased nut intake has been associated with a lower risk of type 2 diabetes and metabolic syndrome; therefore, researchers decided to prospectively examine the association of nut intake with cancer recurrence and mortality in patients with stage III colon cancer receiving adjuvant chemotherapy (Fadelu et al., 2018).

\section{NUT NUTRITION}

Nuts are much more than a nice addition to your cheese platter. Studies have demonstrated that nuts are a valuable source of vegetable protein, monounsaturated fatty acids, vitamin E, folic acid, phytoestrogens, 
and fiber (Gonzalez \& Salas-Salvado, 2007). They play an important role in the reduction of risk for cardiovascular disease and type 2 diabetes (Falasca, Casari, \& Maffucci, 2014). Nuts are essentially a dry fruit with a tough shell around the kernel, and they have a high total fat content, ranging from $46 \%$ in cashews to $76 \%$ in macadamia nuts. Peanuts, by definition, are legumes, rather than traditional tree nuts. Although the consumption of fruits and vegetables is recommended as an important aspect of reducing the risk of cancer, few studies have demonstrated the role of nuts in the prevention of cancer (Gonzalez \& Salas-Salvado, 2007). Emerging data have indicated that inflammation and oxidative stress have a role in the development of cancer; it is possible that the antioxidative and anti-inflammatory properties of nuts and their components may contribute to anticancer activity, as well as confer a cardioprotective effect (Falasca, Casari, \& Maffucci, 2014).

\section{NUT CONSUMPTION AND SURVIVAL IN PATIENTS WITH STAGE III COLON CANCER}

Researchers conducted a prospective, observational study of 826 eligible patients with stage III colon cancer who reported dietary intake on questionnaires during a randomized adjuvant chemotherapy trial (Fadelu et al., 2018). Median follow-up time from completion of the questionnaire was 6.5 years, and during the follow-up period, 199 of the 826 patients demonstrated recurrence of their disease or new primary tumors. One hundred and seventyseven patients from the original cohort died. Patients who consumed more nuts were usually male, consumed more alcohol, and had a lower glycemic load diet, and although the nut users had higher caloric intake, they did not present with a higher body mass index. A significant reduction in recurrence and mortality was associated with increased nut intake after adjusting for other predictors of cancer recurrence. Patients who had diets including two or more servings of nuts per week had an adjusted hazard ratio (HR) for disease-free survival of 0.58 (95\% confidence interval $[\mathrm{CI}]=0.37-0.92$;
$\left.P_{\text {trend }}=.03\right)$. Patients with higher nut intake also had a significant improvement in overall survival (HR, 0.43; 95\% CI = 0.25-0.74; $P_{\text {trend }}=.01$ ). The associations demonstrated in the study appeared to be independent of other predictors of patient outcome, diet, and lifestyle factors (Fadelu et al., 2018). Significantly, an association was not observed with peanuts (which are legumes), as opposed to the positive association determined with tree nuts.

\section{IMPLICATIONS FOR ADVANCED PRACTITIONERS}

Although the study is an observational one, the data are intriguing. Increasing one's consumption of tree nuts would seem to be a relatively simple intervention to implement, and an intervention with a positive outcome in colorectal cancer. Replicating this study is important, and expanding this intervention to other tumor types could be considered. Although the results of this study do not establish causality, they do demonstrate that diet and modifiable lifestyle factors can affect outcomes in patients with colorectal cancer. And as always, advocating for appropriate screening to prevent the development of this common cancer is one of the most critical things we can do to impact this disease. But the next time you add Marcona almonds to your cheese platter, think of the reduction in cardiovascular risk and potential for cancer risk benefits rather than the calorie count. It might be worth it!

\section{References}

American Cancer Society. (2019). Key statistics for colorectal cancer. Retrieved from https://www.cancer.org/cancer/ colon-rectal-cancer/about/key-statistics.html

Fadelu, R., Zhang, S., Niedzwiecki, D., Ye, X., Saltz, L .B., Mayer, R. J.,...Fuchs, C. S. (2018). Nut consumption and survival in patients with stage III colon cancer: Results from CALGB 89803 (Alliance). Journal of Clinical Oncology, 36(11), 1112-1120. https://doi.org/10.1200/ JCO.2017.75.5413

Falasca, M., Casari, I., \& Maffucci, T. (2014). Cancer chemoprevention with nuts. Journal of the National Cancer Institute, 106(9), dju238. https://doi.org/10.1093/jnci/dju238

Gonzalez, A., \& Salas-Salvado, J. (2007). The potential of nuts in the prevention of cancer. British Journal of $\mathrm{Nu}$ trition, 96(suppl 2), S87-S94. https://doi.org/10.1017/ BJN20061868 